\title{
Self-Representation in Social Media During the COVID-19 Pandemic Lockdown
}

\author{
Alexandra Valéria Sándor \\ Doctoral School of Sociology, Eötvös Loránd University, \\ Faculty of Social Sciences, Budapest, Hungary
}

\section{Abstract}

Over the past 15 years, social media has become an integral part of our daily lives, influencing both our informal interactions and professional discourses as well as their structure. From this, one can conclude that social media has its own logic, which includes special norms, strategies and mechanisms (van Dijck \& Poell, 2013). Therefore, it is not surprising that many of the changes affecting society today have been instigated by the use of social media. Two fundamental features of social media are its ability to transcend geographical and cultural boundaries and the role that recipients play when posts are shared, as opposed to traditional models of mass communication. Consequently, these features have led to the rise of the 'infodemic', a term that describes the excessive spread of information and can be used specifically to refer to the dissemination of information about the COVID-19 pandemic on social media platforms (Cinelli et al., 2020). The present study aims to provide a snapshot of how self-representation on social media platforms has changed among Hungarian users during the lockdown period of COVID-19, supported by a brief literature review about the pandemic and its effects on mental health and tested using an online questionnaire to gain deeper insight into social media usage patterns. According to the results of the questionnaire, social media usage and self-representation in social media posts became more frequent during the lockdown. Additionally, symptoms of major depression were more likely among those who shared photos of themselves or their close relations 'daily' or 'multiple times a day' on Messenger, where the frequency of photo-sharing increased the most.

Keywords: COVID-19, self-representation, social media, sociology, social psychology

\section{Introduction}

Over the past 15 years, social media has become an integral part of our daily lives, influencing both our informal interactions and professional discourses as well as their structure. From this, one can conclude that social media has its own logic, which 
includes special norms, strategies and mechanisms (van Dijck \& Poell, 2013). Therefore, it is not surprising that many of the changes affecting society today have been instigated by the increasing usage of social media. Two fundamental features of social media are its ability to transcend geographical and cultural boundaries and the role that recipients play when posts are shared, as opposed to traditional models of mass communication. Consequently, these features have led to the rise of the 'infodemic', a term that describes the excessive spread of information and can be used specifically to refer to the dissemination of information about the COVID-19 pandemic on social media platforms (Cinelli et al., 2020). For example, because this online space allows for immediate response, the number of Internet searches and social media entries in China related to the new coronavirus epidemic peaked 10 to 14 days before the epidemic itself, in terms of the number of new cases (Li et al., 2020). The present study aims to provide a snapshot of how patterns of self-representation on social media platforms have changed among Hungarian users during the lockdown period of COVID-19, with the hypothesis that social media usage became more frequent during the COVID-19 lockdown period, which increased the occurrence of selfrepresentation on various social media platforms.

\section{Literature Review}

In December 2019, news hit the global presses that a novel form of coronavirus had appeared in Wuhan, the most populous city in Central China. It was not difficult to predict that the high population density in Wuhan would be conducive to the transmission of the pathogen via droplet infection. It first spread throughout the rest of Asia, then landed on the shores of other continents, affecting more than a hundred countries in just a few weeks (Remuzzi \& Remuzzi, 2020). In January 2020, the World Health Organization (WHO) declared the epidemic a global public health emergency.

The new coronavirus is extremely contagious, spreads very quickly, and is especially dangerous for those with weakened immune systems. Thus, experts are constantly monitoring new cases and testing those who have come into contact with those who have contracted the disease. Curfew restrictions, lockdowns, quarantine, and physical distancing have been proposed and implemented in many regions of the world (Sanche et al., 2020). For the first time in Europe, these strict restrictions were imposed in Lombardy on 8 March 2020, due to a sharp increase in morbidity in Italy and an almost exponential increase in the number of cases since 21 February, with 9 to $11 \%$ of these cases in need of intensive care (Remuzzi \& Remuzzi, 2020). In Hungary, an epidemiological emergency was declared on 11 March 2020, followed by a lockdown on $28 \mathrm{March}$, initially lasting only two weeks. However, on 9 April, the government extended this lockdown for an indefinite period of time. A gradual easing of restrictions began on 4 May outside the capital city and then on 18 May in Budapest after a significant decrease in the number of new cases. 
The importance of social media during an epidemic has already been recognized once before. During the SARS (severe acute respiratory syndrome) crisis, a coronavirus epidemic which emerged in 2002 and subsided in 2003, social media served as a key means of informing the public and mobilizing public health measures. Furthermore, through spatial and temporal analysis of social media discourses, we can now obtain a comprehensive picture of the epidemiological situation and even generate a realtime map of the spread of the diseases (Yang et al., 2013).

Uncertainties about the future, fears of infection, resource shortages, public health measures that limit personal freedom in unprecedented ways, material losses, and contradictory messages in the media are all emotional burdens associated with the COVID-19 pandemic (Pfefferbaum \& North, 2020). In mental health scholarship, the term 'coronavirus anxiety' was established in the early stages of the pandemic to describe not only the fear of becoming ill but also responses to the difficulties surrounding changing life circumstances. Immense adjustments to the operations of the institutions that govern our daily lives (e.g., schools, workplaces) were being made daily, and the majority of social interactions quickly became digital. Moreover, these changes are not only local but global, affecting entire countries on the level of society. Due to its unique technological features, social media has been used widely to navigate these changes. On social media platforms, users publish virtual selfrepresentations that are influenced by 'real-life' individual and societal changes (Hogan \& Quan-Haase, 2010), while simultaneously learning significant amounts of information about what is happening in 'real life' from the posts of others.

The dire consequences of social media usage during the COVID-19 pandemic are highlighted in the documented case of an Indian man who committed suicide on 12 February 2020. Although his doctor diagnosed him with a different viral infection, the man mistakenly identified his symptoms as indicators of COVID-19 and began obsessively watching videos shared on social media platforms in which Chinese people who had contracted COVID-19 collapsed in public places and were hospitalized against their will (Goyal et al., 2020). While this case may seem like an extreme example, it is certainly thought-provoking in terms of the extent to which users are influenced by the information they find on social media. Researchers observed drastic increases in rates of depression, anxiety, and comorbidities of the two among frequent users of social media during the COVID-19 epidemic (Gao et al., 2020).

\section{Methodology}

To provide a snapshot of the changes in social media usage patterns among Hungarian users from a sociological point of view during the COVID-19 lockdown period, an online questionnaire was created and shared via Facebook and Instagram. Anonymous data collection took place between 22 April and 11 May. During this period, 170 social media users completed the 20 -question questionnaire, answering 
prompts about their basic demographic traits (gender, age, type of settlement, and level of education) and social media usage (which social media platforms they use, what types of content they post, where they post from, and how often), in addition to filling out the PHQ-2 (Patient Health Questionnaire-2) to assess their mental health state. The questionnaire included two open-ended questions to collect the thoughts of participants on social media usage during the lockdown period in the absence of the possibility of a face-to-face interview.

\section{Analysis}

The sample of 170 social media users consisted of 36 men (21.2\%) and 134 women (78.8\%). $2.4 \%$ of respondents were $13-19$ years old, $34.4 \%$ were $20-29$ years old, $30.6 \%$ were $30-39$ years old, $16.5 \%$ were $40-49$ years old, $12.9 \%$ were $50-59$ years old, and 3.5\% were $60-69$ years old. 35.3\% lived in Budapest, $23.5 \%$ in a Hungarian county capital, $23.5 \%$ in another Hungarian city, $12.9 \%$ in a village, and $4.8 \%$ in another country. $43.5 \%$ possessed a university (MA/MSc) degree, $22.4 \%$ had a college (BA/BSc) degree, $18.8 \%$ had a secondary school certificate, $12.9 \%$ had another type of professional qualification, and $2.4 \%$ have had only primary schooling.

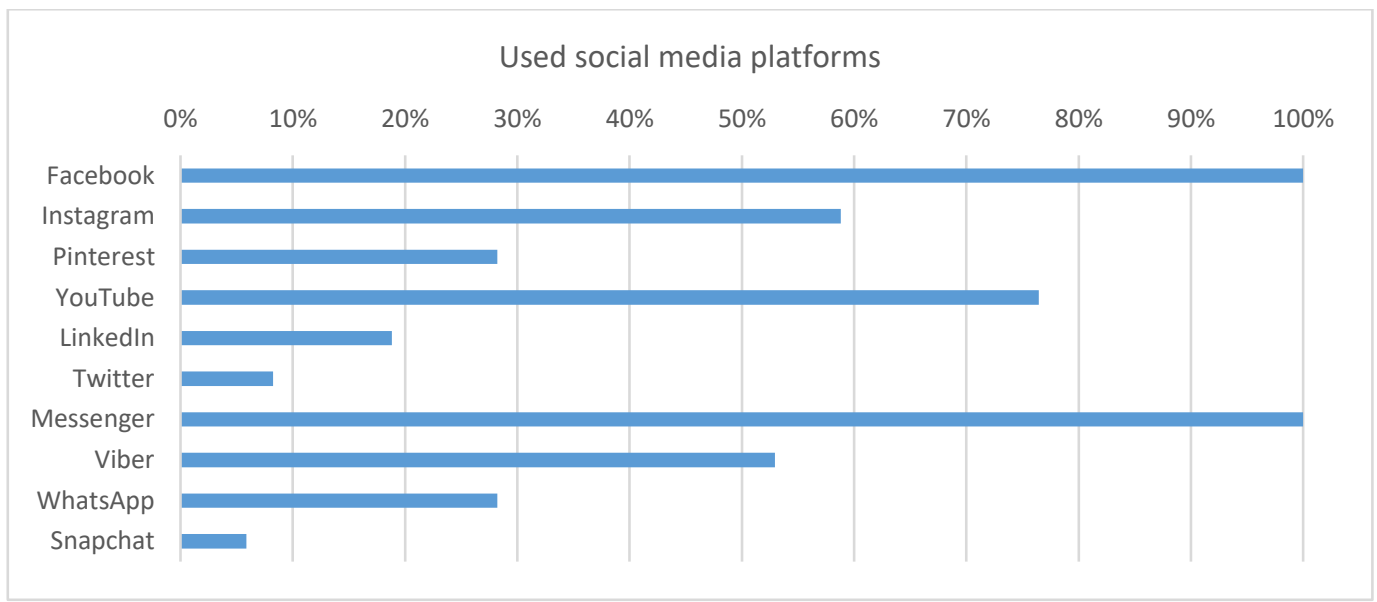

(Figure 1: Usage of social media platforms by participants)

$100 \%$ of the participants reported Facebook and Messenger use. Other social media platforms used included YouTube (76.4\%), Instagram (58.8\%), Viber (52.9\%), WhatsApp (28.2\%), Pinterest (28.2\%), LinkedIn (18.8\%), Twitter (8.2\%), and Snapchat (5.8\%) [Figure 1].

In response to the question, 'How has your total time spent using social media changed during the coronavirus epidemic?', 18.8\% answered 'increased significantly', $35.3 \%$ answered 'increased to some degree', $35.5 \%$ answered 'not changed', and $9.4 \%$ answered 'decreased to some degree' [Figure 2]. As one of the participants wrote, 'I had no choice but to use social media much more frequently because the school of my 
child requires that the teachers communicate with the parents in Facebook groups'. Another responded that they engage in 'one hour of video chat every day with family and friends. I haven't done this before'.

\section{Overall perceived own social media usage during the lockdown

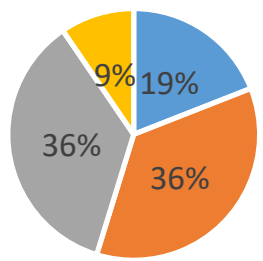 \\ - Increased significantly - Increased in some degree \\ - Not changed $\quad$ Decreased in some degree}

(Figure 2: Overall perceived own social media usage during the lockdown)

The results of the questionnaire displayed a substantial difference between participants' perception of their own changes in social media usage and their impression of these changes in others. $42.4 \%$ of participants thought that others' social media usage 'increased significantly', $45.9 \%$ thought it 'increased to some degree', $5.9 \%$ perceived no change, and $1.2 \%$ perceived that it 'decreased to some degree' [Figure 3].

\section{Perceived social media usage of others during the lockdown}

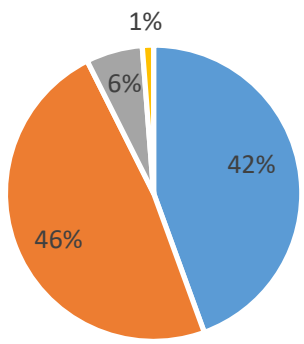

- Increased significantly $\quad$ Increased in some degree $\quad$ Not changed $\quad$ Decreased in some degree

(Figure 3: Perceived social media usage of others during the lockdown)

However, when asked about the change in the numberof reactions received on their photos and videos published on social media, only 3.5\% said these 'increased 
significantly'. 20\% responded 'increased to some degree', 60\% responded 'not changed', $3.5 \%$ responded 'decreased to some degree', and $1.1 \%$ responded 'decreased significantly' [Figure 4]. According to one of the participants, 'pictures of cooking, food, and dogs have become gratuitously high among others'. Another said that 'they have given up the daily posts about where they are and what they are doing, sharing memories instead'.

\section{Reactions received on social media posts during the lockdown \\ - Decreased significantly}

(Figure 4: Reactions received on social media posts during the lockdown)

Thirty-four per cent of participants responded that the amount of the likes and comments they gave to others 'increased to some degree' during the lockdown. Meanwhile, 54.1\% reported no change, and 5.9\% responded that these 'increased significantly' [Figure 5].

Reactions given on social media posts during the lockdown

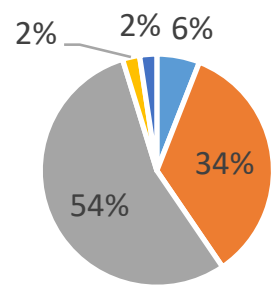

$$
\begin{aligned}
& \text { - Increased significantly } \quad \text { Increased in some degree } \\
& \text { - Not changed } \quad \text { Decreased in some degree } \\
& \text { - Decreased significantly }
\end{aligned}
$$

(Figure 5: Reactions given on social media posts during the lockdown) 
Matrix questions (multiple-choice grids) were used to measure changes in the amount of time spent on each social media platform. Time intervals were presented in the columns ('less than 10 minutes', '10-29 minutes', '30-44 minutes', '45-59 minutes', '60-89 minutes', ' $90-120$ minutes', and 'more than 2 hours'), and the studied social media platforms (Facebook, Instagram, LinkedIn, Twitter, Pinterest, YouTube, Messenger, Viber, WhatsApp, Snapchat) were presented in the rows. Participants responded to this prompt twice: first to record their time usage before the lockdown period, then to record their time usage during the lockdown.

The amount of time spent daily on social media increased remarkably among the participants during the COVID-19 lockdown period. The most notable changes were observed in the case of Facebook, where the most frequently recorded answer (21.1\%) regarding time usage during the lockdown was 'more than 2 hours', followed by ' $10-29$ minutes' and ' $30-44$ minutes' (17.6\% each). In contrast, only $9.4 \%$ spent 'more than 2 hours' on Facebook per day before the pandemic, the most frequently recorded response for time spent on Facebook pre-lockdown being '10-29 minutes'. As a consequence of the pandemic, the number of participants who spend more than 2 hours a day on Facebook increased by $11.8 \%$, thus increasing the frequency of this result by $44.4 \%$ [Figure 5].

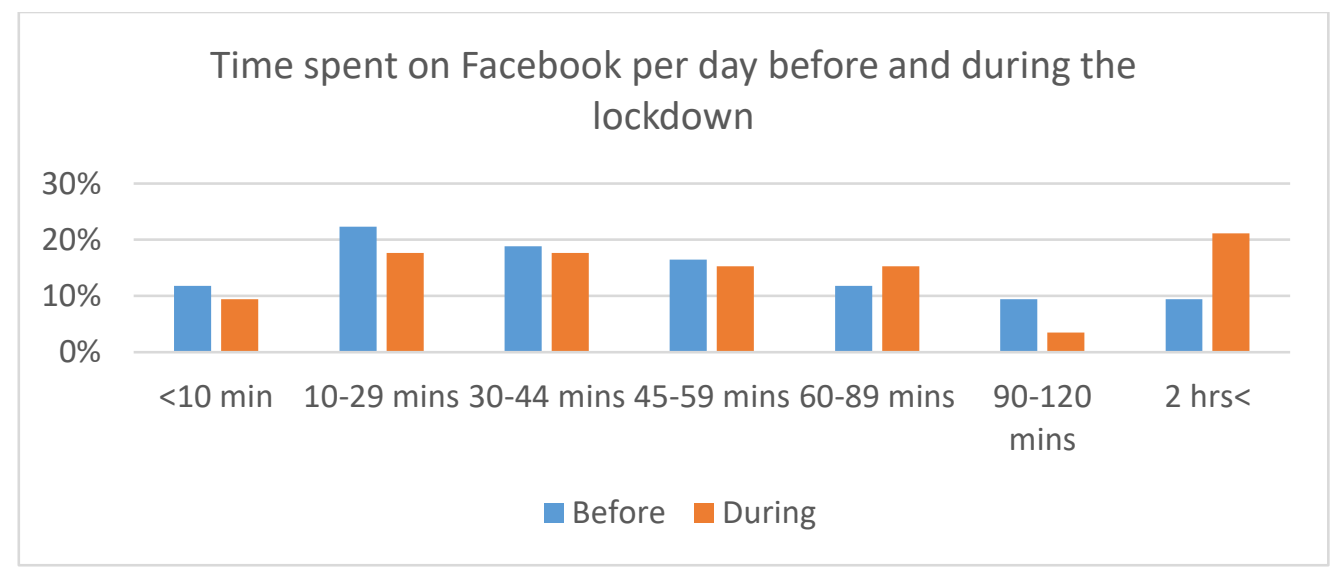

(Figure 5: Time spent on Facebook per day before and during the lockdown)

Messenger also displayed significant changes in time usage. The proportion of participants who answered that they spent 'less than 10 minutes' on Messenger decreased from $21.2 \%$ to $12.9 \%$ during the lockdown, while the proportion of ' $10-29$ minutes' answers increased from $23.5 \%$ to $29.4 \%$ and the proportion of ' $60-89$ minutes' answers increased from $8.2 \%$ to $9.4 \%$. Participants increasingly recorded that they spent '60-89 minutes' (from 8.2\% to 9.4\%), '90-120 minutes' (from 7\% to $8.2 \%$ ), or 'more than 2 hours' (from $8.2 \%$ to $10.6 \%$ ) per day on Messenger [Figure 6]. 
Time spent on Messenger per day before and during the lockdown

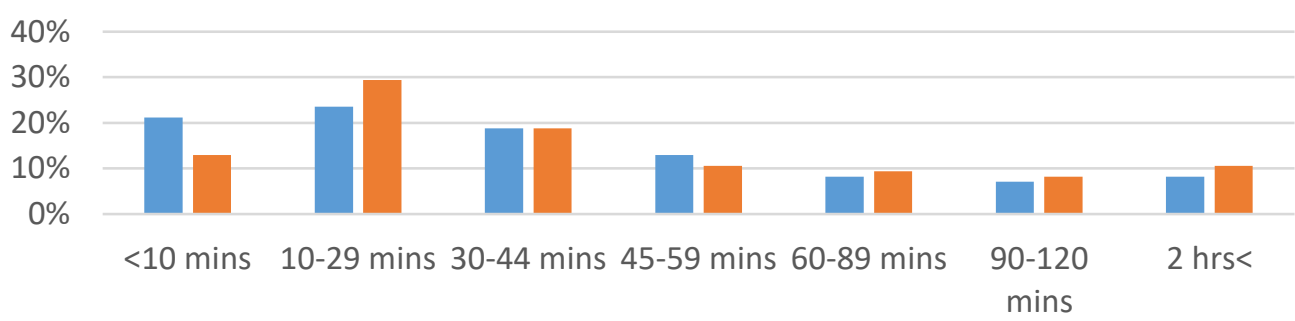

\# Before During

(Figure 6: Time spent on Messenger per day before and during the lockdown)

The third social media platform that displayed considerable changes in terms of time usage was YouTube. The number of participants who spent 'less than 10 minutes' or '10-29 minutes' on Youtube decreased during the lockdown (from $40 \%$ to $34.1 \%$ and $29.4 \%$ to $22.4 \%$, respectively). On the other hand, the number of participants who spent longer amounts of time on YouTube increased. The proportion of participants who recorded '30-44 minutes' spent on the site increased from $9.4 \%$ to $14.1 \%$ during the lockdown, the proportion of '60-89 minutes' responses increased from $4.7 \%$ to $10.6 \%$, and the proportion of ' $90-120$ minutes' responses increased from $1.2 \%$ to $3.5 \%$ [Figure 7].

\section{Time spent on YouTube before and during the lockdown per day}

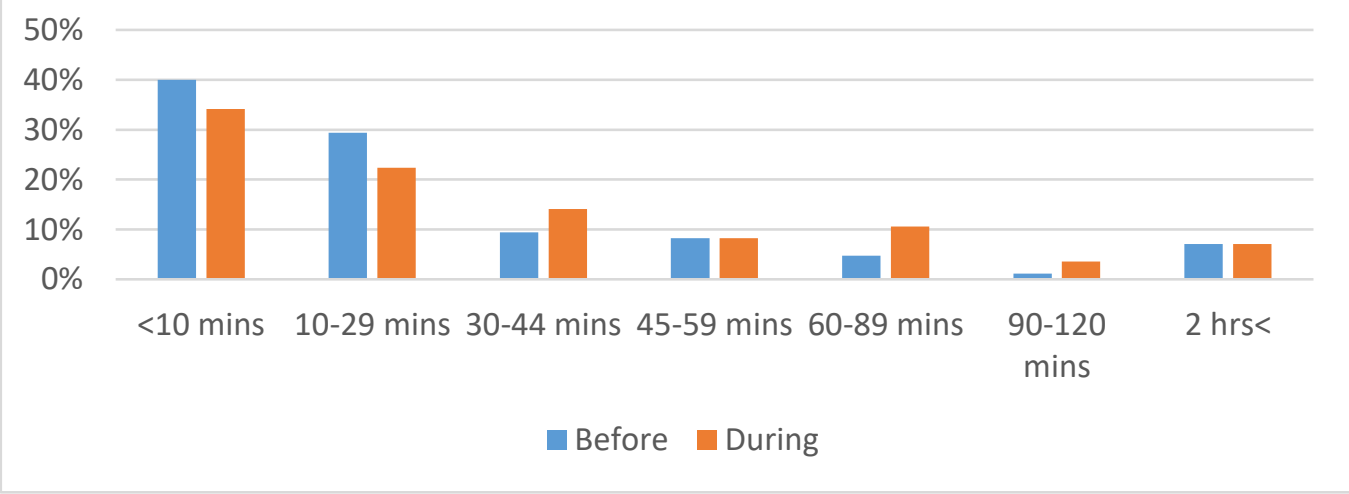

(Figure 7: Time spent on YouTube before and during the lockdown per day)

To measure changes in the intensity of self-representation on social media, participants were asked about the frequency at which they posted photos or videos 
of themselves and close relations before and during the lockdown. According to the data collected, changes were more prominent on social messaging platforms (Messenger, Viber, WhatsApp, and Snapchat) than on social networking sites (Facebook, Instagram, LinkedIn, Twitter, Pinterest, and YouTube).

Almost $18 \%(17.6 \%)$ of participants shared photos and videos of themselves or people closely related to them 'multiple times a day' during the lockdown via Messenger, while only $8.2 \%$ did so before lockdown. Sharing this content 'daily' also became more frequent during the lockdown period, increasing from $3.5 \%$ to $7 \%$ [Figure 8].

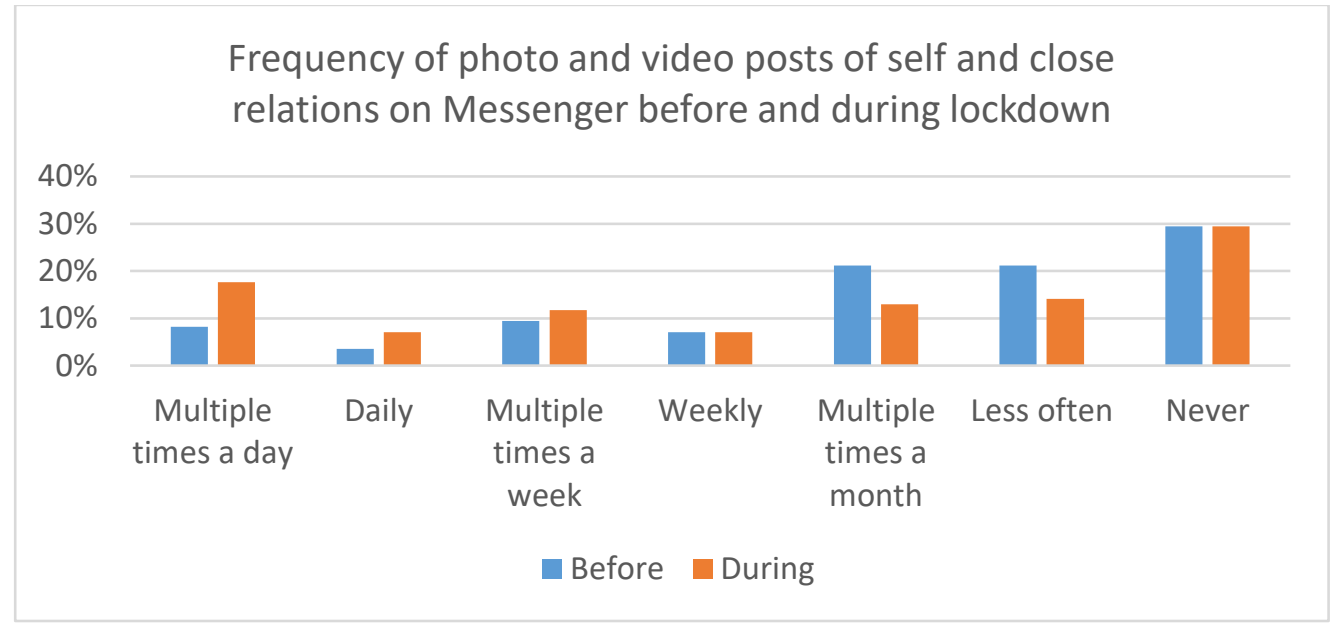

(Figure 8: Frequency of photo and video posts of self and close relations on Messenger before and during the lockdown)

On Viber, 9.4\% of participants shared photos and videos of themselves or close relations 'multiple times a day' during the lockdown, while only $4.7 \%$ did before. The frequency of 'daily' and 'multiple times a week' responses also increased during the lockdown, from $0 \%$ to $2.3 \%$ and from $4.7 \%$ to $5.8 \%$, respectively. 'Weekly' sharing on Viber became more popular as well, increasing from $1.1 \%$ to $4.7 \%$ [Figure 9]. Similarly, sharing photos and videos of oneself and close relations 'multiple times a day' increased from $2.4 \%$ to $3.5 \%$ on Instagram, from $2.4 \%$ to $5.4 \%$ on WhatsApp, and from $3.5 \%$ to $8.2 \%$ on Snapchat. 
Frequency of photo and video posts of self and close relations on Viber before and during lockdown

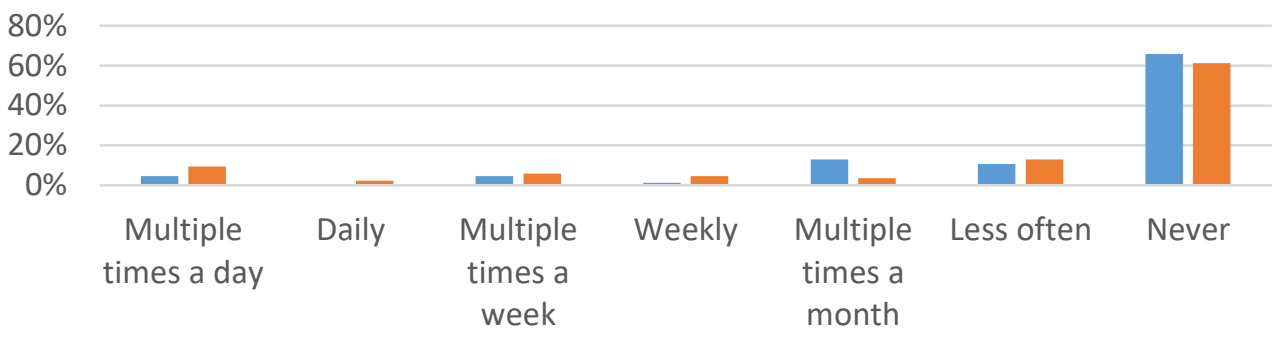

Before During

(Figure 9: Frequency of photo and video posts of self and close relations on Viber before and during the lockdown)

On Facebook, the largest social media platform, sharing photos and videos of oneself and close relations 'multiple times a day' and 'weekly' increased from 3.5\% to 4.7\% and from $1.1 \%$ to $4.7 \%$. However, the ratio of those who 'never' share this type of content increased as well, from $25.9 \%$ to $30.6 \%$ [Figure 10].

Frequency of photo and video posts of self and close relations on Facebook before and during the lockdown

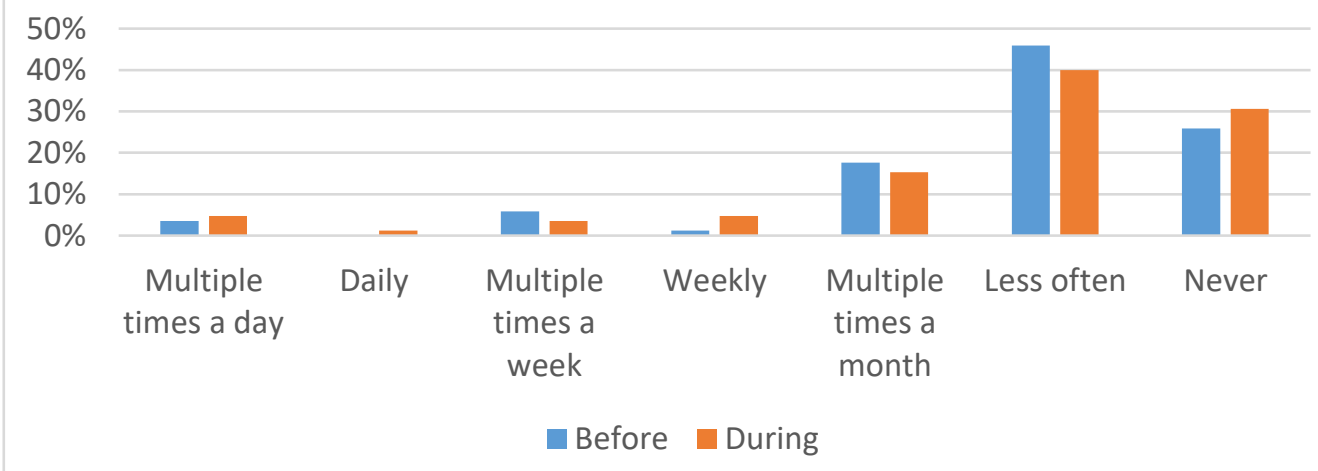

(Figure 10: Frequency of photo and video posts of self and close relations on Facebook before and during the lockdown)

A similar tendency could be observed on Instagram: participants' sharing of photos and videos of themselves and close relations 'multiple times a day' and 'multiple times a week' increased from $2.4 \%$ to $3.5 \%$ and from $4.7 \%$ to $5.9 \%$, while 'never' responses 
increased from $50.6 \%$ to $54.1 \%$ [Figure 11]. As one participant admitted, 'I have much more time to take photos, the process has become more elaborate'.

Frequency of photo and video posts of self and close relations on Instagram before and during the lockdown

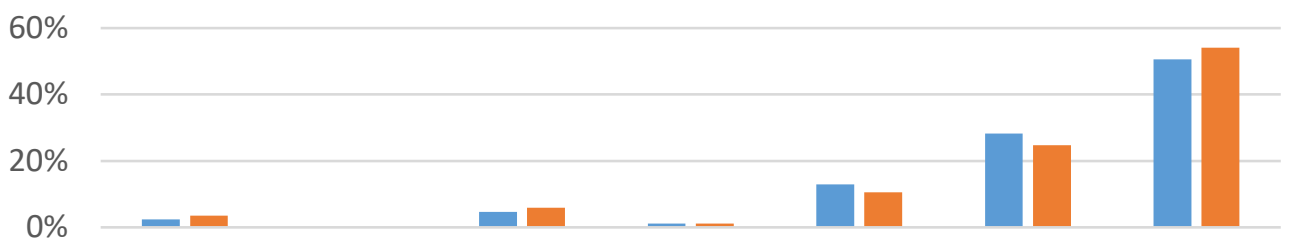

Multiple times a dayaiMultiple times a weldeeNlyltiple times a mbesthoften Never Before During

(Figure 11: Frequency of photo and video posts of self and close relations on Instagram before and during the lockdown)

Regarding the type of self-related photographs on social media during the lockdown, 'selfies' were the most popular among questionnaire participants on all examined social media platforms (Facebook, Instagram, Pinterest, YouTube, LinkedIn, Twitter, Messenger, Viber, WhatsApp, Snapchat) [Figure 12].

Types of self-related photos shared on Facebook during the lockdown

$\begin{array}{lll}\text { - Selfie } & \text { - Portrait } & \text { With others } \\ \text { - At home At work }\end{array}$
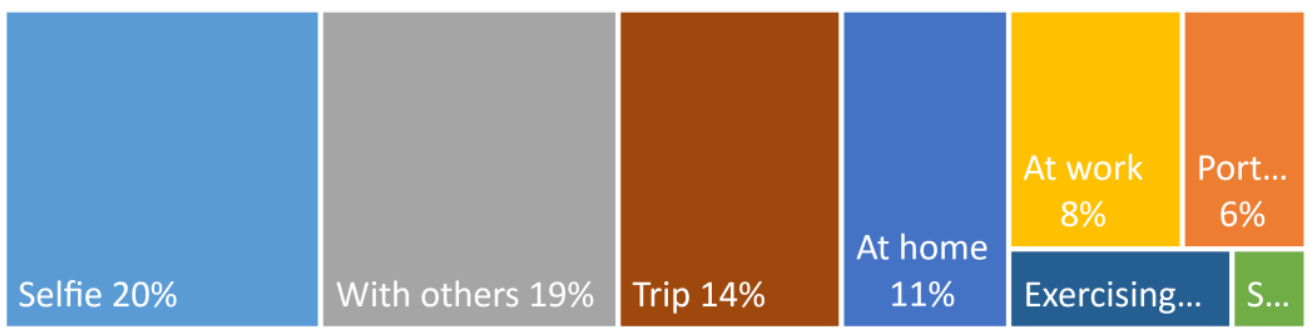

(Figure 12: Types of self-related photos shared on Facebook during the lockdown)

Interestingly, photos taken 'with others' and 'on a trip' were also common during the lockdown. Presumably, these pictures could have been taken before the COVID-19 pandemic, taken with people from the same household, or taken on safe trips where participants adhered to strict social distancing rules [Figure 13]. As one participant wrote, 'I share nice memories'. 
Types of self-related photos shared on Instagram during the lockdown
- Selfie
- Portrait
With others
At work
- At home
- Sexy
- Exercising
- Trip
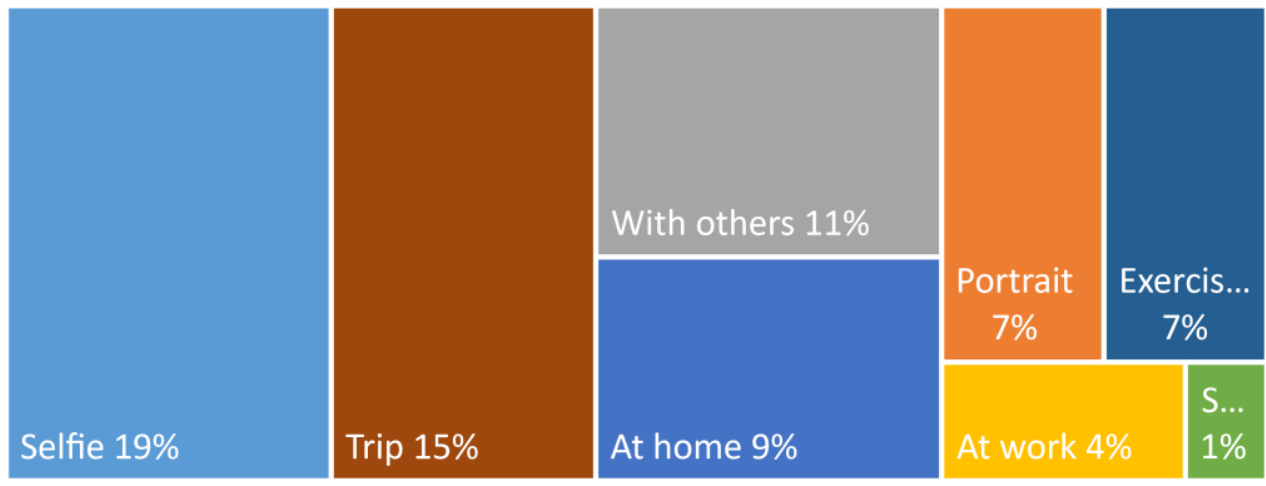

(Figure 13: Types of self-related photos shared on Instagram during the lockdown)

On the other hand, on the more private platform Messenger, the frequency of 'sexy' photographs was higher (3.5\%) than on public sharing sites and even other private messaging platforms such as Viber (2.4\%) [Figure 14].

Types of self-related photos shared on Messenger during the lockdown
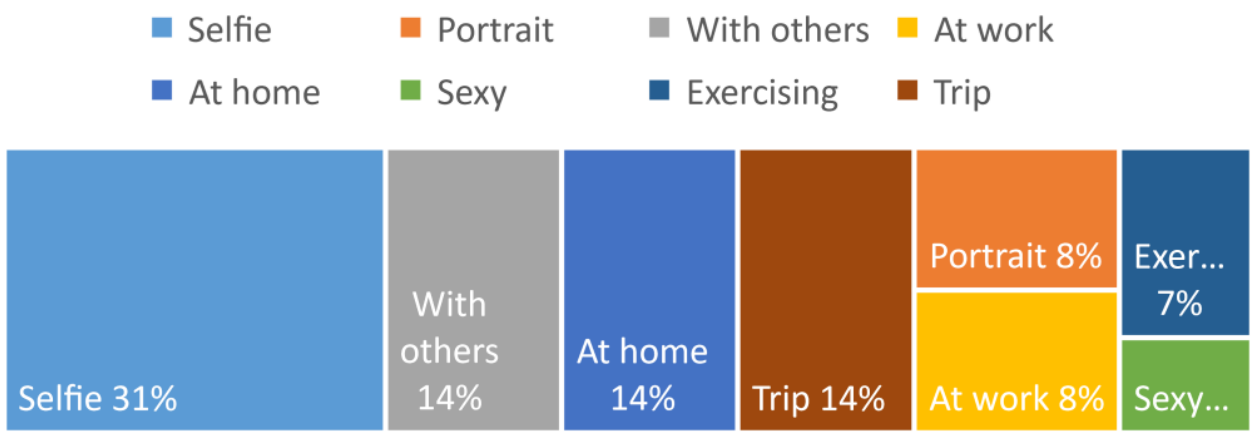

(Figure 14: Types of self-related photos shared on Messenger during the lockdown)

To obtain a basic assessment of the mental health of participants, the Patient Health Questionnaire-2 (PHQ-2) was administered through the question 'Over the last 2 weeks, how often have you been bothered by the following problems?' These problems included 'little interest or pleasure in doing things' and 'feeling down, depressed, or hopeless'. The possible responses were 'not at all' ( 0 points), 'several days' ( 1 point), 'more than half the days' ( 2 points), and 'nearly every day' ( 3 points). 
Final PHQ-2 scores ranged from 0-6. A score of at least 3 indicates the possibility of major depressive disorder and suggests that further examinations are necessary; $16.4 \%$ of participants scored a 3 or higher [Figures 15-17]. However, the likelihood of major depression was higher (19.2\%) among those who shared photos of themselves or their close relations 'multiple times a day' or 'daily' on Messenger, the platform where the frequency of photo-sharing increased the most.

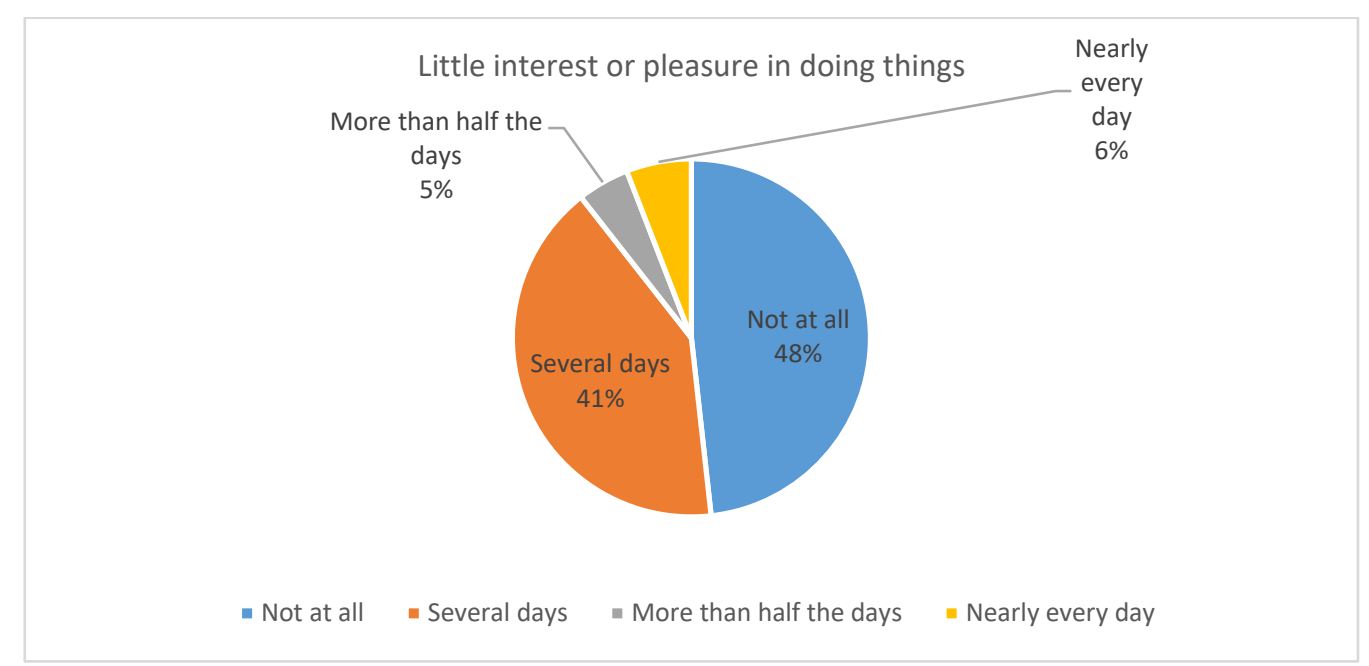

(Figure 15: Answers to PHQ-2 item 'Little interest or pleasure in doing things')

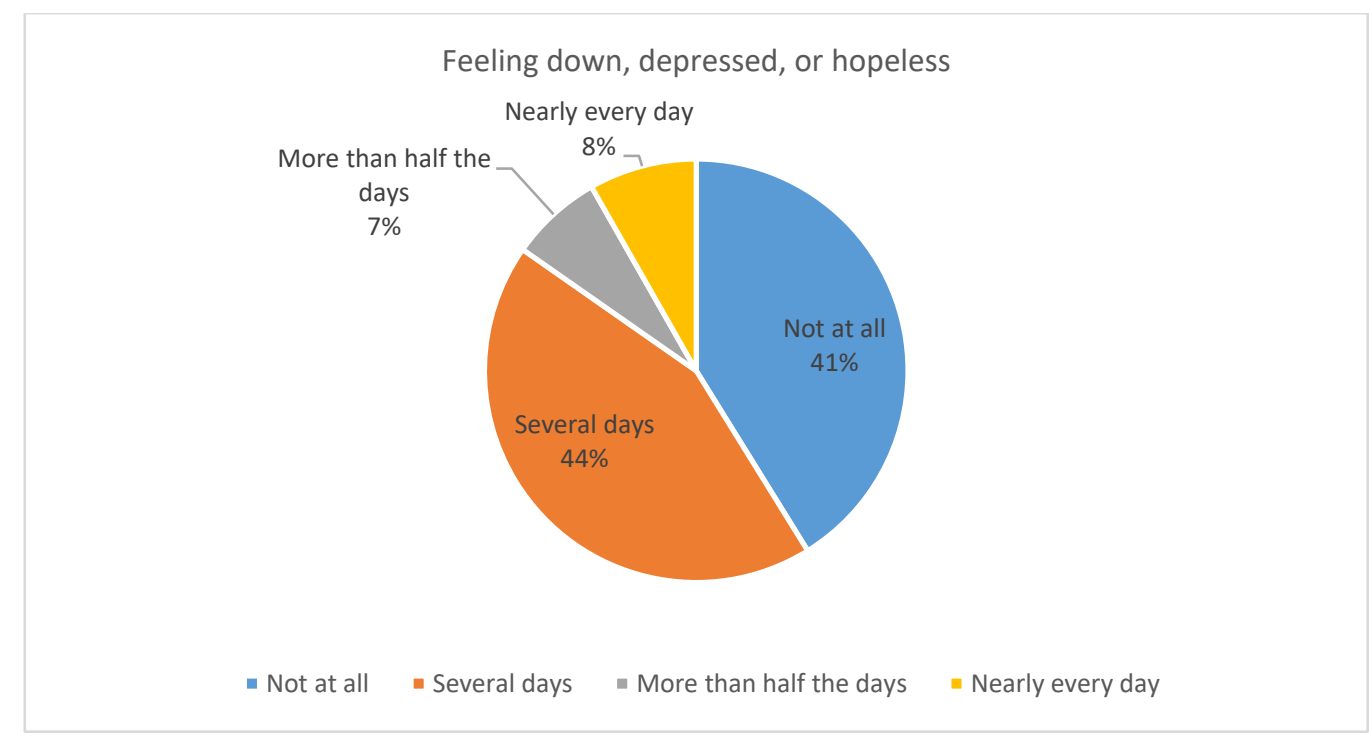

(Figure 16: Answers to PHQ-2 item 'Feeling down, depressed, or hopeless') 


\section{Overall portion of those who are likely to have major} depression

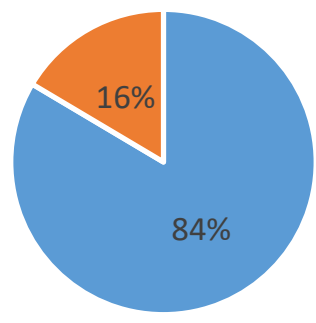

- Not depressed $\quad$ - Likely depressed

(Figure 17: Overall portion of those who are likely to have major depression, according to their PHQ-2 score)

\section{Likelihood of major depression among those who share photos at least once a day on Messenger}

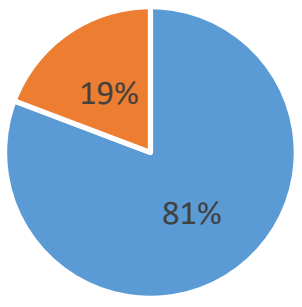

- Not depressed - Likely depressed

(Figure 18: Likelihood of major depression among those who share photos of themselves or their close relations 'multiple times a day' or 'daily' on Messenger, according to their PHQ-2 score)

\section{Conclusion}

The relatively small sample size in this study notwithstanding, the results speak to the ongoing debate about the relationship between social media usage and real-life events through the lens of self-representation. The results of the questionnaire support the hypothesis that social media usage became more frequent during the COVID-19 lockdown period, which increased the occurrence of self-representation on various social media platforms. These findings suggest that the lockdown and social distancing influenced social media users to change their self-representation habits, 
both content-wise and in frequency. The PHQ-2 scores of the participants demonstrate a possible relationship between mental health and the inclination to use social media during the lockdown period. Without a concerted effort to recognise the long-term consequences of online self-representation and social media usage, this phenomenon, among many others, may contribute to the spread of mental health issues as well. Hopefully, these findings will encourage further research on the nature and impact of social media usage and self-representation in social media under special circumstances, for example, unique global or local events, including lockdowns.

\section{Acknowledgements}

The author would like to thank Eötvös Loránd University, Faculty of Social Sciences, Doctoral School of Sociology for the intellectual background, and gives special thanks to supervisor Dr György Csepeli, professor emeritus, head of the Interdisciplinary Social Research doctoral programme.

\section{References}

[1] Cinelli, M., Quattrociocchi, W., Galeazzi, A., Valensise, C. M., Brugnoli, E., Schmidt, A. L., Zola, P., Zollo, F., \& Scala, A. (2020, March). The COVID-19 social media infodemic. arXiv. Retrieved June 5, 2020, from https://arxiv.org/abs/2003.05004

[2] Gao, J., Zheng, P., Jia, Y., Chen, H., Mao, Y., Chen, S., et al. (2020) Mental health problems and social media exposure during COVID-19 outbreak. PLoS ONE, 15(4). https://doi.org/10.1371/journal.pone.0231924

[3] Hogan, B., \& Quan-Haase, A. (2010). Persistence and change in social media. Bulletin of Science, Technology \& Society, 30(5), 309-315. https://doi.org/10.1177/0270467610380012

[4] Lee, S. A. (2020). Coronavirus Anxiety Scale: A brief mental health screener for COVID-19 related anxiety. Death Studies, 44(7), 393-401. https://www.tandfonline.com/doi/full/10.1080/07481187.2020.1748481

[5] Li, C., Chen, L. J., Chen, X., Zhang, M., Pang, C. P., \& Chen, H. (2020). Retrospective analysis of the possibility of predicting the COVID-19 outbreak from Internet searches and social media data, China, 2020. Euro Surveill. https://doi.org/10.2807/1560-7917.ES.2020.25.10.2000199

[6] Pfefferbaum, B. \& North, C. S. (2020, April). Mental health and the COVID19 pandemic. New England Journal of Medicine. Retrieved June 5, 2020, from https://www.nejm.org/doi/full/10.1056/NEJMp2008017

[7] Remuzzi, A. \& Remuzzi, G. (2020, May). COVID-19 and Italy: what next? The Lancet Haematology, 7(5), 365-366. https://www.sciencedirect.com/science/article/pii/S0140673620306279

[8] Sanche, S., Lin, Y., Xu, C., Romero-Severson, E., Hengartner, N., \& Ke, R. (2020). High contagiousness and rapid spread of severe acute respiratory 
syndrome coronavirus 2. Emerging Infectious Diseases, 26(7), 1470-1477. https://dx.doi.org/10.3201/eid2607.200282.

[9] van Dijck, J. \& Poell, T. (2013, August). Understanding social media logic. Media and Communication, 1(1), 2-14. https://ssrn.com/abstract=2309065

[10] Yang, Y. T., Horneffer, M., \& DiLisio, N. (2013). Mining social media and web searches for disease detection. Journal of public health research, 2(1), 17-21. https://doi.org/10.4081/jphr.2013.e4 